\title{
I toponimi nei dizionari bilingui italiano-polacco, polacco-italiano in un contesto diacronico di apprendimento
}

\section{The toponyms in Italian-Polish and Polish-Italian bilingual dictionaries in a diachronic learning context}

\author{
Luca Palmarini \\ Uniwersytet Jagielloński \\ luca.palmarini@uj.edu.pl
}

\begin{abstract}
The purpose of this is to analyze the toponyms present in some Italian-Polish and Polish-Italian bilingual dictionaries. After selecting a "skeleton" of the dictionaries that trace part of the history of the bilingual lexicography in question, we move on to the analysis of the toponyms used in the dictionaries. In a lexicultural context, understood as the transfer of the cultural elements of a given language, we observe the choices made by lexicographers of the different eras and try to understand the reasons, sometimes of a personal nature or dictated by the historical context, or sometimes influenced by the political system in a given country. At the same time, we want to highlight the evolution in the diachronic field that toponyms and their equivalents have had in the dictionaries in question, as well as their value in teaching a particular foreign language.
\end{abstract}

Keywords: Italian-Polish, Polish-Italian bilingual dictionaries, bilingual lexicography, toponyms in bilingual dictionaries, history of Italian language

\section{PREMESSA}

Il presente articolo si propone di analizzare i toponimi presenti in alcuni dizionari bilingui italiano-polacco, polacco-italiano. Dopo aver selezionato un corpus di dizionari che ripercorrono parte della storia della lessicografia bilingue in questione, si passa all'analisi dei toponimi in essi presenti. In un contesto lessi- 
culturale, inteso come trasmissione degli elementi culturali di una data lingua, si osservano le scelte effettuate dai lessicografi delle varie epoche e si cerca di comprenderne le motivazioni, a volte di carattere personale, a volte dettate dal contesto storico in cui si visse, oppure, altre volte ancora, influenzate dal sistema politico vigente in un dato paese. Allo stesso tempo si vuole mettere in evidenza l'evoluzione in ambito diacronico che i toponimi e i loro traducenti hanno avuto nei dizionari in questione, così come il loro valore nell'insegnamento di una data lingua straniera. Spesso si discute se i toponimi vadano lemmatizzati o meno in un dizionario bilingue, se essi siano veramente necessari all'apprendente; ma si dibatte anche sulla loro scelta e funzione, così come sui cambiamenti diacronici cui sono soggetti. In questo contributo ci si propone di osservare la lemmatizzazione dei toponimi in un corpus selezionato di dizionari bilingui italiano-polacco, polaccoitaliano dalle origini (1856) fino ai giorni nostri, con lo scopo di mettere a confronto le eventuali analogie e differenze tra le opere, le scelte dei loro autori e le influenze legate al periodo in cui le opere sono state compilate.

I toponimi dovrebbero essere sempre presenti in un dizionario bilingue, in quanto tali voci non hanno solo un valore di localizzazione (geografico), ma anche un valore onomastico e cognitivo. Secondo alcuni studiosi questi nomi dovrebbero essere inoltre oggetto di una descrizione lessicografica in senso filologico (Chlebda, 1997). La tradizione lessicografica polacca vuole che i toponimi non compaiano nei dizionari monolingui, mentre per quelli bilingui la presenza o meno dei toponimi è stata spesso il risultato di scelte individuali. Esiste chiaramente la consapevolezza del fatto che, se si portassero a lemma tutti i toponimi, la struttura del dizionario crollerebbe e ciò a maggior ragione in un dizionario bilingue (Palmarini, 2018). Si impone, dunque, una selezione dei lemmi, dando ampio spazio alle aree geografiche delle due comunità linguistiche a cui il dizionario bilingue è destinato. È proprio grazie a lemmi come i toponimi che il dizionario diventa il luogo adatto in cui realizzare ciò che nei suoi studi sulla lessicultura Robert Galisson definisce pragmatique lexiculturelle (Galisson, 1999, p. 478) ${ }^{1}$. Infatti, scegliendo un toponimo al posto di un altro, una sua variante invece di un'altra, proponendo traducenti di toponimi differenti rispetto ad altri dizionari, si offrono all'utente informazioni di carattere lessiculturale, ovvero attraverso le parole si trasmette la cultura di una lingua e della sua comunità, concetti basilari per chi apprende una lingua straniera. Per chi, invece, studia la lessicografia in chiave diacronica, si hanno importanti informazioni del periodo storico in cui il dizionario è stato stilato, ma anche una certa attualizzazione, in un'analisi contrastiva, dei cambiamenti avvenuti ${ }^{2}$.

\footnotetext{
${ }^{1}$ Per l'interculturalità nei dizionari crf p.es. : Gallison, R. (1991). De la langue à la culture par les mots, Paris: CLE International; Szende, T. (2003) (a cura di). Les écarts culturels dans les dictionnaires bilingues, Paris: H. Champion; Laurian, A.M. (2004). Dictionnaire bilingue et interculturalité, Berne: Peter Lang.

${ }^{2}$ In un dizionario bilingue non solo i toponimi, ma anche gli avvenimenti storici, le festività, il sistema scolastico, la politica, l'economia, il diritto, le unità di misura, la valuta, la storia letteraria, le
} 
Dall'analisi del corpus selezionato si ricaveranno informazioni di carattere lessicale e culturale, a conferma del fatto che il dizionario bilingue è anche lo specchio di due culture e dei loro tempi. Si cercherà, inoltre, di trovare conferma del fatto che anche i toponimi nei dizionari bilingui sono utili all'apprendimento di una lingua straniera.

\section{UN BREVE EXCURSUS STORICO SUI DIZIONARI DEL CORPUS}

La lessicografia bilingue italiano-polacca, polacco-italiana è relativamente giovane. Il primo dizionario di questo tipo fu pubblicato nel 1856 con il titolo di Dokładny słownik włosko-polski, seguito, un anno più tardi, dalla seconda parte, Dokładny słownik polsko-włoski. Si tratta di un dizionario a carattere enciclopedico, ricco di tecnicismi, citazioni letterarie da entrambe le letterature delle lingue in questione, proverbi, modi di dire, esempi di utilizzo, a volte anche fonti etimologiche. L'autore, Erazm Rykaczewski, erudito polacco in esilio, lessicografo e autore di grammatiche polacche, proponeva con esso uno strumento di studio della lingua italiana per i suoi connazionali (in particolare quelli costretti all'esilio) che volessero leggere i classici della letteratura italiana in originale. L'autore stesso si guadagnava da vivere insegnando privatamente il francese e l'italiano ma anche il polacco.

Il secondo dizionario del corpus, datato 1860, è anch'esso in due volumi, intitolati rispettivamente Słownik podręczny włosko-polski e Słownik podręczny polskowłoski. Si tratta di un'opera lessicografica con una microstruttura scarna, nella quale l'autore, Ignacy Pląskowski, si limita nella maggior parte dei casi a proporre un semplice schema di entrate e traducenti.

Nel 1913 viene pubblicato in un unico volumetto il dizionario dell'italiano Fortunato Giannini - frate scolopio che risiedeva a Cracovia - dal titolo Stownik włosko-polski, polsko-włoski. Questo dizionario avrà un buon successo editoriale, sia per il suo formato tascabile sia per il carattere didattico e comunicativo che propone interessanti esempi di utilizzo. Fino agli anni Cinquanta, se si escludono i dizionarietti del Secondo Corpo D'Armata Polacco in Italia, non vengono pubblicati altri dizionari bilingui italiano-polacco, polacco-italiano. Con il disgelo tra Italia e Polonia (l'anno di svolta per quest'ultima è il 1956) riprendono i rapporti diplomatici e culturali; nel giro di pochi anni vengono realizzati addirittura due dizionari bilingui, entrambi stampati dalla stessa casa editrice, la PWN di Varsavia, ognuno dei quali pubblicato in due volumi. La prima coppia di dizionari in ordine cronologico è Stownik włosko-polski (1960), di Stanisław Soja e Zbigniew Zawadzki, e Słownik polsko-włoski (1961) di Soja, Celeste Zawadzka e Zawadzki. Si tratta di due volumetti di formato tascabile che presentano un aspetto comunicativo e di-

istituzioni, il cibo, l'abbigliamento (insieme costituiscono i realia), contribuiscono a mettere in evidenza le differenze tra le due lingue e culture, veicolando un corpus di natura sociolinguistica. 
dattico. La seconda coppia di volumi, ad opera di Wojciech Meisels, docente di lingua italiana presso l'Università Jagellonica di Cracovia, viene pubblicata con i seguenti titoli: Podręczny słownik włosko-polski (1964) e Podręczny słownik polsko-włoski (1970), quest'ultimo volume uscito postumo. È un'opera che, rispetto alla precedente, propone un lemmario con molte voci di carattere scientifico e tecnico, altre volte di carattere storico (Palmarini, 2016). Il fine didattico-comunicativo è presente, ma ad esso si affiancano anche quello filologico e della lettura dei classici italiani, andando incontro al modello di studi universitari allora vigente. Entrambi i dizionari domineranno il mercato per tutta la seconda metà del XX secolo e saranno oggetto di successive edizioni rivedute. Nel caso di Meisels si avrà anche un'edizione ampliata (1986 e successive), portata a termine da un gruppo di docenti universitari. Al tempo stesso, il dizionario sarà epurato dalle citazioni d'autore e dalla nomenclatura scientifica di piante ed animali, che lo rendevano troppo scientifico, appesantendone il contenuto. Grazie a questa successiva edizione riveduta il Meisels divenne il più popolare dizionario bilingue italiano-polacco, polaccoitaliano dell'ultimo ventennio del Novecento, importante strumento per chi imparava l'italiano in quel periodo negli atenei polacchi e non solo.

Infine, nel corpus vengono inclusi anche due dizionari del primo decennio del XXI secolo; il primo, uscito in due volumi, Uniwersalny słownik polsko-włoski (2004), e Uniwersalny słownik włosko-polski (2008), è stato curato da Maria Podracka. Il secondo dizionario in questione, dal titolo Wielki słownik włosko-polski - Grande dizionario italiano-polacco, è stato pubblicato in cinque volumi con il patrocinio dell'Accademia della Crusca, curato da diversi italianisti e polonisti, italiani e polacchi ${ }^{3}$. Costituisce un'opera ambiziosa, il più corposo e ricco dizionario bilingue italiano-polacco, polacco-italiano finora realizzato.

Lo scopo del lavoro è quello di analizzare la presenza dei toponimi, di comprendere le scelte effettuate dai vari lessicografi, scelte che possono essere personali o dettate proprio dal cambiamento dei tempi e come esse possano influire sull'apprendimento della lingua straniera.

\section{IL PRIMO DIZIONARIO BILINGUE ITALIANO-POLACCO, POLACCO-ITALIANO (1856 E 1857)}

Il dizionario di Rykaczewski in due volumi, Dokładny słownik włosko-polski e Dokładny słownik polsko-włoski, si presenta corposo, erudito e di carattere

\footnotetext{
${ }^{3}$ Pubblicato da Wiedza Powszechna in cinque volumi dal 2001 al 2010; volume 1 (2001), A-E, H. Cieśla, E. Jamrozik, R. Kłos; volume 2 (2002), F-O, H. Cieśla, E. Jamrozik, J. Sikora Penazzi; volume 3 (2006), P-Sezzo, H. Cieśla, E Jamrozik, J. Łopieńska, J. Sikora Penazzi; volume 4 (2010), Sf-Z, J. Sikora Penazzi. A essi si aggiunge un quinto volume (2001) che contiene regole grammaticali, verbi, nomi propri di persona e toponimi, quindi verrà analizzato e inserito nella bibliografia di riferimento.
} 
enciclopedico (Sosnowski, 2005). Come accennato, sono presenti numerose citazioni d'autore, proverbi ed esempi di utilizzo. L'opera presenta una relativa bidirezionalità: è stata infatti creata anche per favorire il mantenimento della lingua e della cultura polacche in un momento storico in cui la Polonia è spartita tra Russia, Prussia e Austria (Palmarini, 2018). Il dizionario di Rykaczewski non si rivela comunque essere adatto alla produzione di testi, ma si propone soprattutto ai polacchi colti per la lettura dei classici italiani; leggendo queste opere essi imparavano l'italiano con un metodo filologico-traduttivo (Sosnowski, 2005).

Dopo questa breve presentazione delle caratteristiche del dizionario, può stupire il fatto che in questa opera lessicografica i toponimi siano quasi del tutto assenti. Questa carenza suscita ancora maggior stupore se si considera che una delle fonti principali di Rykaczewski per la parte polacco-italiano è il dizionario tedescopolacco di Mrongovius (1837) che di toponimi ne registra invece centinaia. Chiaramente bisogna sottolineare che, dal punto di vista geografico e culturale, il mondo polacco e tedesco di allora erano a stretto contatto, spesso i due elementi etnicoculturali si sovrapponevano, quindi una maggiore presenza di lemmi di tal genere in un dizionario bilingue tedesco-polacco risulta giustificata se non persino naturale, in quanto, sebbene Mrongovius proponga anche toponimi di continenti e città di tutto il mondo, il suo dizionario rispecchia soprattutto il microcosmo bilingue a cui lo stesso lessicografo apparteneva, ovvero le regioni della Varmia, Masuria e Pomerania (Nowowiejski, 2012). Rykaczewski, invece, segnala pochissimi toponimi che non lemmatizza in una rubrica a parte, ma inserisce direttamente nel lemmario comune. In Dokładny słownik włosko-polski non si riscontrano nomi di continenti, né idronimi e limnonimi. È presente un solo oronimo: “ALPE, pl. - PI. s.f. Alpy". Riguardo ai coronimi si registra solo la presenza di "UNGHERIA, s.f. Wegry.", "POLONIA, s.f. Polska." e "TURCHÌA, s.f. Turcya". Nel caso del lemma Polonia la scelta lessiculturale risulta naturale, in quello di Ungheria gli elementi che possono indurre a comprendere la scelta di Rykaczewski di inserirlo in un dizionario quasi del tutto privo di altri nomi di indicazione geografica sono due: la vicinanza geografica, storica e culturale tra i due paesi ${ }^{4}$ e il fatto che Ungheria sia l'esonimo di Węgry, quindi forse anche una scelta di carattere linguistico. Si annota ancora l'interessante entrata Quirinale: "QUIRINALE, ad. e s. m. góra Kwirynalska, jedna z gór rzymskich, na której Romolus miat kościót, dziś Monte Cavallo, z pałacem papieskim". In questo caso tale lemmatizzazione ci suggerisce come il dizionario assuma un carattere enciclopedico e di come questa voce presenti l'elemento lessiculturale legato al ruolo storico politico che il palazzo stava svolgendo in quel momento storico.

\footnotetext{
${ }^{4}$ I due popoli sono accomunati da una fratellanza storico-culturale. Anche i rapporti con la Turchia furono intensi, soprattutto di carattere guerresco e di ambasceria.

5 "Monte del Quirinale, uno dei colli di Roma, sul quale Romolo aveva la chiesa, oggi Monte Cavallo con il Palazzo Papale" (Le traduzioni dal polacco sono opera dell'autore dell'articolo).
} 
Il secondo volume, Doktadny stownik polsko-wtoski, presenta qualche toponimo in più rispetto al primo, ma anche qui si tratta di una quantità intorno alla decina. A lemma troviamo i seguenti coronimi: "FRANCYA, YI, s.f. Francia", "GRECYA, YI, s.f. Grecia", NIEMCY, MIEC, s.pl. geog. Allemagna, Germania", "WŁOCHY, $\mathrm{CH}$, s.pl Italia.". Interessante è osservare come, eccetto Wlochy, questi lemmi siano annidati ${ }^{6}$ all'interno di altre voci di radice comune, rispettando un ordine alfabetico.

Ancora si registra la presenza dell'idronimo "WISŁA, Y. S., Vistola" nel quale l'autore annida l'aggettivo "WIŚLANY, A, E, di Vistola". Interessante, sempre nel dizionario polacco-italiano, è il poleonimo Kraków che viene presentato così: "KRAKÓW, OWA, s.m. Geog. Cracovia. Nie za jeden dzień Kraków zbudowany $^{7}$, prov. Roma non fu fatta ad un tratto, al primo colpo non cade l'albero". Oltre alle marche d'uso, sempre presenti in Rykaczewski, appare l'abbreviazione della marca categoriale "Geog.", ma ciò non è una costante. Il lemma Kraków assume qui una duplice funzione, non solo di localizzazione, ma anche simbolica (Czerny, 2011), in quanto il ruolo di capitale storica di Cracovia viene confermato da un proverbio e il suo corrispondente in italiano in cui la città polacca viene accostata a Roma.

Un altro poleonimo presente è appunto "RZYM, U, s.m. Roma". In tutte le voci dal polacco Rykaczewski indica la terminazione del genitivo (qui è "u"), continuando la tradizione lessicografica polacca inaugurata dal lessicografo Samuel Bogumił Linde e mostrandosi attento all'aiuto nelle regole grammaticali. Si tratta anche di una conferma del desiderio di Rykaczewski di proporre il suo dizionario anche come strumento per l'apprendimento della lingua polacca ${ }^{8}$.

L'autore del primo dizionario bilingue polacco-italiano, italiano-polacco non mostra un grande interesse verso i toponimi, lemmatizzandone solo alcuni per ognuna delle due parti di cui è composto il dizionario. Le voci registrate sono troppo poche per stabilire quale sia stato il criterio di scelta del lessicografo (probabilmente non ne esiste alcuno), ma senza dubbio la componente lessiculturale cui appartiene l'autore è di facile intuizione. Nel caso dell'oronimo Alpe e dell'idronomo Vistola, non si tratta solo di due puri elementi geografici, ma anche dei simboli di due nazioni, diventati anche tematica letteraria, così come il ruolo storico di Cracovia.

\footnotetext{
${ }^{6}$ Il concetto dell' "annidare", da me esposto in precedenza (Palmarini, 2018), consiste nell'inserire nella microstruttura di un lemma altre voci che hanno una radice comune. All'interno di un lemma principale, scelto secondo criteri alfabetici o di frequenza, vengono annidati altri sottolemmi.

7 "Cracovia non è stata costruita in un giorno".

${ }^{8}$ Nelle sue opere grammaticali e lessicografiche Rykaczewski guardava anche ai polacchi nati all'estero che non dovevano perdere il contatto con la lingua madre polacca.
} 


\section{IL DIZIONARIO BILINGUE SLOWNIK PODRECCZNY WŁOSKO-POLSKI DI IGNACY PLĄSKOWSKI (1860)}

Assai diversa si presenta la situazione dei toponimi nel Słownik podręczny włosko-polski di Ignacy Pląskowski, in cui essi sono lemmatizzati in una rubrica a parte. Il dizionario bilingue di Pląskowski, in due volumi, si presenta come un'opera lessicografica assai modesta, dove la microstruttura dei lemmi spesso è ridotta al minimo, con nella maggior parte uno o due traducenti e le marche categoriali, mentre gli esempi di utilizzo sono quasi completamente assenti. Uno dei pochi punti a favore di questo dizionario è proprio la rubrichetta (presente solo nel volume italiano-polacco) dal titolo Wyrazy geograficzne [Espressioni geografiche], di circa 6 pagine. Tra le caratteristiche generali si osserva che, rispettando l'ortografia polacca ottocentesca, l'autore riporta coerentemente la Y dopo le consonanti nei toponimi di stati, regioni e città: Aleksandrya, Alzacya, Austrya, Szwajcarya, Francya, Grecya, Kroacya, Hollandya, Irlandya, Islandya, Szkocya, Szwecya, Turcya, Rossya. In alcuni casi nei traducenti polacchi la forma latina viene preferita a quella polacca, "Transilvania, $f$ Transylwania", mentre il nome polacco di questa regione è Siedmiogród ${ }^{9}$. Si osserva come le informazioni grammaticali siano ridotte al genere del lemma: "Brasile, $m$ Brazylia", "Portogallo, $m$ Portugalia". A volte è presente il corrispondente perifrastico: è il caso di "Ardenne (selve) $f$. $P l$ Lasy ardeńskie". Dopo la Grande Guerra il toponimo assumerà una funzione simbolica e si tralascerà il perifrastico (infatti nei dizionari successivi si riscontra semplicemente Ardenne). Spesso sono presenti ulteriori informazioni: "Reno, m. Ren (rzeka)", "Ródano, m Rodan (rzeka)", "Rodi, f. Rodus (wyspa)". Questa situazione non è però una costante: "Danùbbio, $m$ Dunaj”. In molti casi sono riportate varianti di lemma: "Fiorenza, Firenze, $f$. Florencja", "Gand, Gàndavo, $m$ Gandawa", “Giena, Jena, $f$ Jena”, “Gotlandia, Gotia, $f$ Gotlandia”. Si tratta di varianti storiche, ma bisogna purtroppo sottolineare che l'utente non viene informato su quale delle due forme sia desueta e quale invece sia attuale. Nel caso dei nomi composti in cui è presente l'aggettivo, Pląskowski, sempre rispettando le regole ortografiche polacche di allora, riporta entrambi i termini con le lettere maiuscole, ma ciò in italiano non avviene: "Paesi bassi, $m$. pl. Niderlandy", oppure "Paesi bassi uniti, $m$. pl. Zjednoczone Niderlandy." Sono presenti alcuni toponimi che rispecchiano un'antica denominazione italiana attinta direttamente dal latino, per esempio "Biponto, $m$. pl. Dwamosty", forma desueta di Zweibrücken (Bipontum in latino). Il lemma in questione è la conferma dell'usanza linguistica, ancora in voga nel XIX secolo, di tradurre i nomi delle città dal latino. Nel caso di "Leópoli $f$. Lwów" l'autore non propone l'esonimo tedesco di Lemberg, allora anch'esso nome ufficiale della città, ma il traducente polacco "Lwów", sottolineandone l'appartenenza culturale alla

\footnotetext{
${ }^{9}$ Così infatti lemmatizzeranno i suoi successori.
} 
Polonia e non all'Austria di cui allora politicamente Leopoli faceva parte, rispettando anche il binomio lemma della lingua di partenza e traducente della lingua d'arrivo, al di là della geografia politica del momento.

Il panorama toponomastico di Pląskowski si presenta vario: si va dai corpora acquae, seppur in numero esiguo - sono lemmatizzati sotto la voce mare, "Mare Atlántico, m. Morze Atlantyckie", "Mar Caspio, o di Sala, m. Morze Kaspijskie" a idronimi come "O'dera, $f$ Odra", "Meno, $m$. Men (rzeka).", a isole, "Rodi $f$ Rodus (wyspa).”, a oronimi "Táuro, $m$. Taurus (góra).”, per poi continuare con coronimi, "Friuli, $f$. Fryol.", "Podolia, $f$. Podole.", "Podláchia, $f$. Podlasie." e numerosi poleonimi "Passavia, $f$. Passau", "Stetino ${ }^{10}, m$. Szczecin".

Rispetto al resto del dizionario, il materiale toponomastico di indicazione geografica proposto da Pląskowski si presenta ricco e ci trasmette il ritratto di un lessicografo che sembra sapersi orientare nel mondo a lui coevo, così come nella geografia storica. In un'ottica lessiculturale ho avuto modo di notare già in precedenza (Palmarini, 2018) come il mondo toponomastico del dizionario di Pląskowski sia incentrato soprattutto su nomi geografici riguardanti l'Europa centrale. Tale osservazione sembra essere ovvia, ma la presenza di toponimi tedeschi è nettamente dominante, grazie alla lemmatizzazione di centri di medie o piccole dimensioni come per esempio "Servesta, $f$. Zerbst.", "Silvaduca, $f$. Hercogenbusz.", "Smalcalda, $f$. Smalkaldya.", Sneberga, $f$. Szneeberg.", "Sundgovia, f. Sundgowia". Proprio questo fatto $\mathrm{mi}$ ha indotto a ritenere che una delle fonti dell'opera di Pląskowski potesse essere un dizionario tedesco dell'epoca e dopo alcuni confronti con opere lessicografiche tedesche del periodo ho trovato conferma a tale ipotesi nel Nuovo dizionario portatile italiano-tedesco (Bottarelli, Barretti, Jageman, Aldeung, 1843). In seguito a un'analisi di tipo contrastivo si evince chiaramente che Pląskowski ha trasferito tutto il lemmario di questo dizionario tedesco nella sua opera. $\mathrm{Su}$ qualunque punto ci si soffermi, si osserva che il lemmario è identico, anche nelle varianti. Eccone un esempio:

Tab. 1. Confronto delle voci presenti nei due dizionari, da Salisburgo a Sshelda

\begin{tabular}{|l|l|}
\hline \multicolumn{1}{|c|}{ Nuovo dizionario portatile italiano-tedesco } & \multicolumn{1}{c|}{ Słownik podręczny włosko-polski } \\
\hline Salisburgo & Salisburgo \\
\hline Salonichi, Tessalonica & Salonichi, Tessalonica \\
\hline Samogizia & Samogizia \\
\hline Samotracia & Samotracia \\
\hline San Bernardo il Grande, il piccolo & San Bernardo il Grande, il piccolo \\
\hline San Domingo & San Domingo \\
\hline San Gallo & San Gallo \\
\hline
\end{tabular}

\footnotetext{
${ }^{10}$ Riportato con una sola $\mathrm{T}$.
} 


\begin{tabular}{|l|l|}
\hline \multicolumn{1}{|c|}{ Nuovo dizionario portatile italiano-tedesco } & \multicolumn{2}{c|}{ Słownik podręczny włosko-polski } \\
\hline Sardegna & Sardegna \\
\hline Sardo & Sardo \\
\hline Sassónia & Sassónia \\
\hline Sassóne & Sassóne \\
\hline Savoja & Savoja \\
\hline Savojardo & Savojardo \\
\hline Scaffusa & Scaffusa \\
\hline Scandinavia & Scandinavia \\
\hline Scánia & Scánia \\
\hline Sshelda & Sshelda \\
\hline
\end{tabular}

La rubrica dei toponimi presente nel dizionario bilingue di Pląskowski offre interessanti spunti per un'analisi da cui si evincono numerosi elementi lessiculturali. Il fatto che il lemmario sia frutto di una completa trasposizione da un altro dizionario non rende meno interessante l'analisi dei toponimi, delle soluzioni proposte e dei traducenti scelti. A livello d'apprendimento, però, l'apporto si riduce a un mero binomio lemma-traducente.

\section{SŁOWNIK WŁOSKO-POLSKI, POLSKO-WŁOSKI DI FORTUNATO GIANNINI (1913)}

Il dizionario Stownik włosko-polski, polsko-włoski di Giannini, con finalità didattiche generali maggiori rispetto ai precedenti, propone i toponimi tra i lemmi comuni. Sono presenti tutte le regioni italiane, mentre in Pląskowski ve n'è solo una rappresentanza. Anche i poleonimi riguardanti l'Italia sono numerosi; se ne citano alcuni: "Firénze (-ce) $f$ Florencya $f$ ", "Nápoli $f$ Neapol $m$ ", "Miláno $f$ Medyolan $m$ ", ma ve ne sono molti altri anche di svariate città minori come per esempio Siracúsa. Si nota come lo scolopio tramite l'accento proponga la pronuncia per i polacchi, mettendo in evidenza il fine didattico presente nell'opera. Dal punto di vista fonetico polacco si osserva che l'autore rispetta ancora la vecchia trascrizione con la Y già segnalata in Pląskowski. Se ci si addentra nella preparazione culturale di Giannini, italiano, religioso e autore di grammatiche ricche di testi estratti dal mondo religioso e da quello classico, si possono comprendere alcune scelte da lui effettuate riguardo ai nomi geografici. Infatti, si trovano a lemma toponimi riguardanti le sacre scritture, per esempio Gérico, Carmélo, Babéle, Babilónia, Gólgota e altri attinenti al mondo classico, Parnáso, Olímpo, Mesopotámia, Núbia, Pompéi. Allo stesso tempo, si evince la centralità dello Stato italiano: oltre a molte città d'Italia si registrano anche toponimi specifici, inusuali per un dizionario di tali dimensioni, 
come: Valtellína, Caríddi, Elba, Vesúvio, Moncenísio, Monte Biánco, ma di importante carattere storico.

L'opera di Giannini è un chiaro esempio di come un dizionario dei primi del Novecento rispecchi ancora le scelte individuali di un singolo autore. Le scelte dello scolopio in tale ambito sono anche legate ai testi classici e di carattere religioso presenti nelle sue grammatiche (e, di conseguenza, nelle sue lezioni), per le quali il dizionario si propone come ottimo supporto didattico.

\section{SŁOWNIK WŁOSKO-POLSKI DI SOJA E ZAWADZKI, E SŁOWNIK POLSKO-WŁOSKI DI SOJA, ZAWADZKA E ZAWADZKI (1960 E 1961)}

In entrambe le parti del primo dizionario bilingue italiano-polacco, polaccoitaliano del secondo dopoguerra è presente una rubrica, dal titolo Nazwy geograficzne, nomi geografici, che occupa cinque pagine nella parte italiano-polacco e altrettante nella parte polacco-italiano, si tratta quasi di un ribaltamento (Marello, 1989). In entrambe le rubriche il titolo è prima in polacco, scritto in maiuscolo, di seguito in italiano, ma in minuscolo, a sottolineare la funzione unidirezionale, secondo la definizione sempre di Marello (1989), dell'opera, realizzata soprattutto per la comunità linguistica polacca.

Il dizionario in questione presenta interessanti aspetti lessiculturali, in quanto in esso sono presenti i cambiamenti dei confini e dei sistemi politici, cambiamenti che hanno avuto luogo in seguito alla divisione dell'Europa in due blocchi. Infatti, la nomenclatura imposta nei paesi a regime comunista fa sentire la sua ingombrante presenza anche nei dizionari bilingui. Nel dizionario le voci dei toponimi che definiscono i paesi del blocco comunista sono lemmatizzate con un sistema a doppia entrata: "Albania $f$ Albania" e "Repubblica Popolare dell'Albania, Albańska Republika Ludowa", "Bulgaria, $f$ Bułgaria" cui segue nella microstruttura "Bułgarska Republika Ludowa Repubblica Popolare di Bulgaria" e ancora "Vietnam $f$ Wietnam", "Wietnamska Republika Ludowo Demokratyczna Repubblica Popolare-Democratica del Vietnam". La stessa situazione si riscontra nella parte polaccoitaliana: "Czechosłowacja $f$ Cecoslovacchia $f$ " e "Czechosłowacka Republika Socjalistyczna Repubblica Socialista Cecoslovacca” oppure "Jugosławia $f$ Jugoslavia f", Jugosłowiańska Federacyjna Republika Ludowa Repubblica Federale Jugoslava". Il dizionario, però, non riporta a lemma le singole repubbliche sovietiche nella loro appellazione ufficiale, ma solo la denominazione classica. Infatti, ad esempio, leggiamo "Białoruś $f$ Bielorussia $f$ ".

Sono presenti nomi propri di continenti; sempre nella parte polacco-italiano si trovano "Ameryka Południowa America del sud" e "Ameryka Północna America del nord", senza alcuna marca categoriale anticipata nel lemma precedente "Ame- 
ryka $f$ America $f$ ". Si riscontrano coronimi, ma anche oronimi: "Apeniny plt Appennini $m \mathrm{pl}$ ". Sempre riguardo agli oronimi si osserva come questo dizionario abbia accolto la centralità geografica dello Stato polacco, lemmatizzando tutte le catene montuose di questo paese: "Beskidy plt Beschidi $m$ pl", "Góry Świętokrzyskie Monti di Santa Croce", "Karpaty plt Carpazi $m$ pl", "Sudety plt Sudeti $m$ pl", "Tatry plt Tatra $m p l$ ". In entrambe le parti sono presenti alcune regioni italiane, mentre si riscontra la presenza di tutte le regioni storiche polacche, come per esempio "Slesia $f$ Śląsk $m$ (Alta, Bassa - Górny, Dolny Śląsk)".

Per quanto concerne sempre le scelte influenzate dai cambiamenti dei sistemi, si osserva come nel lemmario venga incluso un fiume che dal punto di vista delle dimensioni non è ritenuto tra i più importanti della Polonia. Infatti, nella parte polacco-italiano si legge: "Nysa $f$ Nissa $f$ (fiume)" $"$. Questo idronimo è entrato nella terminologia politica italiana all'interno dell'espressione 'linea Oder-Neisse', intesa come nuova linea di confine, assumendo una certa importanza geopolitica. Come traducente non viene però proposta la forma tedesca 'Neisse', usata nella diffusa espressione 'Oder-Neisse'. Gli autori erano con ogni probabilità portati ad abbandonare la terminologia tedesca e in questo caso a riscoprire l'idronimo latineggiante 'Nissa' che si ritrova in testi ben più antichi ${ }^{12}$. La presenza di questo idronimo a discapito di altri come i fiumi Bug o Warta, ben più lunghi e storicamente più importanti della Nysa, avvalora l'interesse del sistema comunista per la conferma dell'appartenenza alla Polonia dei territori tolti ai tedeschi, del nuovo confine. Questa voce non si limita a svolgere una funzione localizzativa, di indice, ma trasmette un ulteriore significato legato al luogo (Czerny, 2001). Lo stesso lemma si riscontra nella parte italiano-polacco: "Nissa $f$ Nysa $f$ ".

Ora si osservino i poleonimi delle tre grandi città tedesche diventate polacche dopo il 1945: "Szczecin, $m$ Szczecin, Stettino", "Wroclaw, $m$ Wrocław, Breslavia", "Gdańsk, $m$ Gdańsk, Danzica $f$ ". Oltre a una microstruttura coerente, si nota subito come nei traducenti al primo posto venga proposto il toponimo polacco e solo in seguito il nome tradizionale storico, non rispettando né l'ordine alfabetico, né quello di frequenza. Nella lingua italiana, infatti, ancora oggi si continuano a usare i termini Stettino, Breslavia, Danzica, ma gli autori, scegliendo di posizionare al primo posto dei traducenti gli stessi termini, sembrano voler trasmettere il messaggio che ora le città sono polacche e il loro nome in polacco dovrebbe essere adottato anche dalla lingua italiana, scalzando quelli storici di chiara provenienza germanica. I toponimi del Soja-Zawadzki e del Soja-Zawadzki-Zawadzka confermano come anche l'insegnamento di parole straniere in Polonia fosse sottoposto alla dottrina socialista.

\footnotetext{
${ }^{11}$ Esiste anche una città con tale poleonimo.

${ }^{12}$ Per esempio nell'opera Geografia Cioè Descrittione Universale Della Terra si legge: "Nissa fiume della Lusatia” (Tolomeo, 1598, p. 63).
} 


\section{PODRĘCZNY SŁOWNIK WŁOSKO-POLSKI E PODRĘCZNY SŁOWNIK POLSKO-WŁOSKI DI WOJCIECH MEISELS (1964-1970)}

Anche il dizionario bilingue di Wojciech Meisels presenta una rubrica di toponimi a sé stante, dal titolo, in entrambi i volumi, Nomi geografici nazwy geograficzne, dalla cui analisi si possono ricavare interessanti osservazioni. Meisels, allo stesso modo del dizionario precedente, lemmatizza i nomi dei paesi del blocco comunista con una doppia nomenclatura, prima quella classica a cui segue quella socialista: "Bulgaria $f$ Bulgaria; Repubblica Popolare della - Bułgarska Republika Ludowa”. A differenza del dizionario di Soja-Zawadzki e Soja-Zawadzki-Zawadzka, il lessicografo cracoviano registra anche i toponimi delle repubbliche facenti parte dell'Unione Sovietica: "Bielorussia $f$ Białoruś $f$; Repubblica Socialista Sovietica di - Białoruska Republika Radziecka", e ancora "Georgia $f$ Gruzja $f$; Repubblica Socialista della - Gruzińska Socjalistyczna Republika Radziecka". Sempre in un'ottica contrastiva nell'ambito del sistema socialista, si può osservare quali siano le scelte di Meisels riguardo alle città passate dalla Germania alla Polonia dopo il secondo conflitto mondiale: nella parte italiano-polacco Stettino e Danzica sono a lemma, ma si riscontrano anche le forme polacche Gdansk e Wroclaw, curiosamente private delle rispettive lettere polacche ń e ł. Il lessicografo sembra qui guardare alle nuove forme, con la proposta di trasferirle alla lingua italiana come prestiti integrati, senza però abbandonare quelle classiche. Anche nella parte polacco-italiano l'autore non sembra effettuare una scelta coerente: "Gdańsk a $m$ Gdańsk, Danzica $f$ ", "Szczecin - a $m$ Stettino $f$ ", "Wroclaw - ia, Wroclaw $f$ ". Sempre nella parte polacco-italiano si evidenzia anche la presenza dell'acronimo "ZSRR $m$ URSS", inteso anch'esso come un toponimo la cui funzione simbolica è molto forte, al pari di USA.

Nella sua vita Meisels è stato anche studioso di letteratura classica, soprattutto latina. Si osserva ora se questo elemento lessiculturale si sia potuto riflettere nella scelta delle entrate. A lemma troviamo voci come "Al(I)emagna", $f$ Niemcy $p l$ ", "Alvernia $f$ Owernia $f$ ", "Asfaltide (lago) Morze martwe", "Benaco $m$ Jezioro Garda", "Lario $m$ Jezioro Como". Si tratta di nomi storici, latineggianti, che nel caso dei due limnonimi Lario e Benaco sono ancora ufficiali, ma desueti, mentre nel caso del coronimo Allemagna e del limnonimo Asfaltide sono utilizzati esclusivamente in ambito storico. Meisels questo lo sapeva, ma nel dizionario purtroppo tralascia di informare l'utente. Lo stesso caso si riscontra nel desueto "Paludi Pontini (sic!) (Pontyjskie błota)" cui già nel secondo dopoguerra si preferiva 'Agro Pontino', e nell'esonimo "Regiomonte, $f$ Królewiec $m$ "13.

Sono presenti le regioni storiche polacche, per esempio Podlachia, Pomerania, Masovia, Varmia, Silesia Slesia, così come molte regioni italiane. Richiamandoci

\footnotetext{
${ }^{13}$ Dal 1945 Kaliningrad.
} 
al dizionario di Pląskowski che riportava Ardenne con il perifrastico Selve, qui si osserva come Meisels propenda per un'opzione più moderna: "Ardenne $f p l$ Ardeny $f$ ", che trasmette la precedentemente citata funzione simbolica.

Dopo queste brevi osservazioni si evince come Meisels nella scelta dei lemmi subisca influenze dai suoi studi classici, sebbene ometta di segnalare il valore storico di alcune voci. Allo stesso tempo l'autore si adatta alla nuova nomenclatura socialista, ma non elimina completamente le voci storiche (vedi Stettino e Danzica), risultando più elastico, probabilmente in conseguenza di una timida apertura del regime.

\section{WIELKI SŁOWNIK WŁOSKO-POLSKI}

Agli inizi del XXI secolo vengono pubblicati due importanti lavori lessicografici nei quali compaiono toponimi. Tra il 2001 e il 2002 escono i primi due volumi di Wielki stownik włosko-polski Grande dizionario italiano-polacco a cura di Hanna Cieśla, Elżbieta Jamrozik e Radosław Kłos. Nel 2001, oltre al primo volume che comprende i lemmi dalla lettera A alla E, esce un volumetto, a cura di Elżbieta Jamrozik e Ilona Łopieńska, che raccoglie regole grammaticali, verbi irregolari, nomi propri di persona e di luogo. La rubrica dal titolo Nazwy geograficzne, Nomi geografici è ad opera di Ilona Łopieńska, conta 17 pagine, è dall'italiano al polacco, si presenta con parecchie varianti - come nel caso di "Timbuctù, Tombuctù, Tombouctou $f$ Timbuktu $n$ " - e rimandi, alcuni interessanti, come "Rụssia Bianca $z o b$. Bielorụssia". Anche nei toponimi (come nel resto del dizionario) viene segnalato dove cade l'accento, elemento assai importante per l'apprendente polacco che nel caso di tali lemmi è interessato alla forma grafica e alla pronuncia ${ }^{14}$. Interessante è il caso dell'entrata Ucraina: "Ucraịna, Ucrạina dawn. Ucrạina $f$ Ukraina $f$. In Italia si sentono entrambe le pronunce, il Wielki stownik chiarisce che a quella tradizionale si è affiancata quella più filologica, Ucraịna. Esistono anche interessanti varianti storiche: "Treviso, dawn. Trevigi $f$ Treviso $n$ ". Dal punto di vista italo-polacco si registra una buona presenza di città italiane, ma anche delle più importanti città polacche: Cracovia, Varsavia, Stettino, Breslavia e quelle storiche di Leopoli e Vilna. In quest'ultimo lemma si registra anche la variante Vilnius, a conferma di come gli autori della rubrica siano attenti ai cambiamenti storicofilologici in atto, in quanto il lemma Vilna è ormai desueto, sostituito da quello di una città oggi capitale della Lituania e diversa dalla Vilna polacca.

14 "Dal confronto del sistema fonetico italiano con quello polacco risulta ad esempio che, siccome i lessemi polacchi sono nella stragrande maggioranza piani, diventa necessario segnalare all'utente le parole italiane accentate in modo diverso che sulla penultima sillaba" (Jamrozik, 2006). 


\section{UNIWERSALNY SŁOWNIK WŁOSKO-POLSKI E UNIWERSALNY SŁOWNIK POLSKO-WŁOSKI (2004-2008)}

Il dizionario, a cura di Katarzyna Podracka, viene pubblicato in due parti, la prima, Uniwersalny stownik wtosko-polski, esce nel 2004, mentre la seconda, Uniwersalny stownik polsko-wtoski, nel 2008. La parte italiano-polacco, oltre ai lemmi classici, comprende varie rubriche, tra cui una dal titolo "Włoskie nazwy geograficzne Luoghi d'Italia". Le voci lemmatizzate propongono alcune informazioni che danno loro un carattere tra enciclopedico ed etimologico. Ecco come per esempio viene lemmatizzata la voce Veneto:

Veneto -Wenecja Euganejska (region). Toponim pochodzi od nazwy dawnych mieszkańców tego obszaru nazywanych przez Rzymian Veneti, zaś przez Greków Henetói i wywodzi się od indoeuropejskiego wen - 'desiderare' (= pożądać, życzyć sobie). Zgodnie $\mathrm{z}$ tą teorią Veneti byliby zatem ludem pożądanym ${ }^{15}$.

Si tratta di un interessante esempio di come un dizionario, strumento per la didattica, attraverso elementi lessiculturali possa trasmettere non solo informazioni linguistiche ma anche culturali, necessarie all'apprendimento di una lingua straniera e dei diversi aspetti della vita sociale che essa veicola.

Il secondo volume, l'Uniwersalny stownik polsko-wtoski, contiene invece una rubrica dal titolo "Nazwy geograficzne, Nomi geografici". In essa sono presenti interessanti divisioni in categorie dei toponimi: 1) Kontynenty, Continenti 2) Państwa, Kraje / Stati, Paesi 3) Miasta, Miejscowości / Città, Località 4) Regiony, Prowincje, Stany / Regioni, Province, Stati 5) Góry - Łańcuchy i Szczyty górskie / Monti - Catene e Vette 6) Morza, Oceany / Mari, Oceani 7) Rzeki / Fiumi 8) Jeziora / Laghi 9) Wyspy / Isole. Si tratta di una divisione i cui titoli sono adattati pensando all'utente non specialista di linguistica e per questo motivo non presentano i nomi ufficiali della classificazione dei toponimi (idronimi, limnonimi, coronimi, etc.). Tutti i laghi sono preceduti dal lessema jezioro [lago] o Wielkie jezioro [grande lago]. Un'eccezione è il limnonimo Balaton che appare in solitario. Si tratta di un importante elemento lessiculturale che si spiega con lo storico ruolo turistico che questo lago ha avuto negli anni della Repubblica Popolare di Polonia. Il lago è diventato così conosciuto che in polacco non ha più bisogno del perifrastico jezioro. L'elemento 'patriottico' che nel Soja-Zawadzki e nel Soja-Zawadzka-Zawadzki puntava alla sostituzione dei toponimi tedeschi o di origine tedesca oggi non ha più ragion d'essere. Troviamo, per esempio, "Odra $s f$. Oder", "Uznam $s m$. Usedom". Per un confronto si osserva ora il lemma Nysa, in cui si legge: "Nysa $s f$ Lużycka, Nysa $f$ Lużycka/Neisse". Oltre al fatto che gli autori propongono Nysa con il

${ }^{15}$ Trad. It. "Veneto - Venezia (regione). Il toponimo deriva dal nome degli antichi abitanti di questa regione, chiamati dai romani Veneti e dai greci Henetói, e nasce dall'indoeuropeo wen 'desiderare'. Secondo questa teoria i veneti sarebbero allora stati un popolo desiderato". 
perifrastico Lużycka (per differenziarlo da un altro fiume, la Nysa Kłodzka), si nota come sia presente anche la forma tedesca che effettivamente nella geopolitica italiana ha prevalso nettamente fino ad oggi. Nel caso del fiume Odra il traducente italiano è la forma tedesca Oder. In questo moderno dizionario abbiamo la conferma del fatto che lo storico influsso della cultura tedesca sui toponimi in territori oggi compresi nella Polonia occidentale rimane ancora attuale.

L'Uniwersalny stownik ribadisce come alcune località abbiano assunto una certa importanza storica (spesso legata a eventi purtroppo negativi) e siano diventate oggetto di lemmatizzazione: "Oświęcim, sm. Auschwitz", "Dachau, sn. inv. Dachau", "Czarnobyl sm. Černobyl”. Si riscontrano anche informazioni di carattere storico; sono presenti i nomi di alcune repubbliche storiche: "Republika $s f$ Cisalpińska (St.) Repubblica $f$ Cisalpina", "Republika $s f$ Genueńska (st.) Repubblica $f$ di Genova", "Republika $s f$ Liguryjska (st.) Repubblica $f$ Ligure", "Republika $s f$ Neapolitańska (st.) Repubblica $f$ Partenopea", "Republika $s f$ Wenecka (st.) Repubblica di Venezia". Il carattere enciclopedico che in questo caso assume il dizionario trova conferma nell'importante abbreviazione "st." (storico).

Dal punto di vista della scelta del modello linguistico, un interessante esempio è dato dalla voce San Remo. L'Uniwersalny stownik propone: "San Remo sn. inv. San Remo / Sanremo $f$ ". In polacco il nome della famosa città rivierasca è scritto separato, come anche nel caso del toponimo 'Monte Cassino'. Gli autori propongono due traducenti. Il nome della città è l'abbreviazione fonetica di San Romolo, vescovo genovese. Le stesse parole San Remo erano presenti in tutte le mappe della Repubblica di Genova, del Regno di Sardegna e poi del Regno d'Italia, ma in alcuni testi locali esisteva anche la forma univerbale (Rossi, 1867). La dizione ufficiale Sanremo è comparsa nei documenti del periodo fascista. Infine, all'inizio del XXI secolo è stata definitivamente scelta la forma univerbale. Tale cambiamento, se non recepito, porta a seri problemi informatici, per esempio nello svolgimento di elezioni, nelle mappe satellitari e nella raccolta dei dati Istat. Il dizionario propone, giustamente, entrambe le varianti.

La rubrica dei nomi geografici di entrambi i dizionari pubblicati nei primi anni del XXI secolo ne sottolinea la modernità, ma allo stesso tempo evidenzia anche lo sviluppo della classificazione dei toponimi nella lessicografia bilingue con una sempre più netta divisione tra toponimi attuali e desueti (con marche categoriali dawn. e st.), un numero maggiore di varianti, dove cade l'accento (Wielki stownik) e i sottogruppi geografici (Uniwersalny).

\section{CONCLUSIONI}

La presenza dei toponimi in un dizionario bilingue si è rivelata essere un fattore importante per l'apporto di elementi lessiculturali al dizionario stesso. Si è confermato come sia possibile inserire in un'opera di questo genere nomi propri di luogo, 
ma anche di come il microcosmo lessiculturale che ne consegue sia complesso e in continuo sviluppo. Si è avuto modo di appurare come le scelte dei toponimi siano state, più di altre voci, una scelta che nella prima fase della lessicografia bilingue italiano-polacca, polacco-italiana (1856-1946) era appannaggio del singolo lessicografo, di come, invece, durante il periodo comunista siano stati introdotti profondi cambiamenti dovuti all'affermazione di nuovi assetti geopolitici con il tentativo di emarginare la toponomastica tedesca nelle regioni della sua influenza storica, e, infine, come nel caso dell'Uniwersalny, di come un dizionario moderno sappia dividere i vari campi interni ai toponimi, facilitando all'utente l'accesso alle informazioni. I toponimi racchiudono informazioni lessiculturali di grande interesse per l'apprendimento, che rispecchiano la cultura e il percorso storico di entrambe le comunità linguistiche. 


\section{BIBLIOGRAFIA}

Dizionari del corpus analizzato

Giannini, F. (1913). Stownik włosko-polski, polsko-włoski. Berlin: Neufeld-Henius.

Łopieńska, I. (2001). Nazwy geograficzne. In E. Jamrozik, I. Łopieńska, (ed.), Wielki słownik włosko-polski: podstawy gramatyki języka włoskiego, czasowniki nieregularne, nazwy geograficzne, imiona, postacie historyczne $i$ mitologiczne, skróty i skrótowce włoskie, oznakowanie włoskich prowincji (pp. 169-187). Warszawa: Wiedza Powszechna.

Meisels, W. (1964). Podręczny słownik polsko-włoski. Warszawa: Państwowe Wydawnictwo Wiedza Powszechna.

Meisels, W. (1970). Podręczny stownik włosko-polski. Warszawa: Państwowe Wydawnictwo Wiedza Powszechna.

Pląskowski, I. (1860). Słownik podręczny włosko-polski. Warszawa: Edizione propria, presso libreria G. Sennewald.

Pląskowski, I. (1860). Słownik podręczny polsko-włoski. Warszawa Edizione propria, persso libreria G. Sennewald.

Podracka, M.K. (a cura di) (2004). Uniwersalny stownik wtosko-polski. Warszawa: Rea.

Podracka, M.K. (a cura di) (2008). Uniwersalny stownik polsko-wtoski. Warszawa: Rea.

Rykaczewski, E. (1856). Doktadny stownik wtosko polski. Berlin: Behr.

Rykaczewski, E. (1857). Dokładny stownik polsko włoski. Berlin: Behr.

Soja, S., Zawadzka, C., Zawadzki, Z. (1961). Słownik polsko-włoski. Warszawa: Wiedza Powszechna.

Soja, S., Zawadzki, Z. (1960). Stownik włosko-polski. Warszawa: Wiedza Powszechna.

\section{Opere di riferimento}

Chlebda, W. (1997). Zarys polskiej geografii mentalnej. Przeglad Humanistyczny, 3, 81-94.

Czerny, A. (2011). Teoria nazw geograficznych. Warszawa: PAN.

Galisson, R. (1999). La pragmatique lexiculturelle pour accéder autrement, à une autre culture, par un autre lexique. Études de Linguistique Appliquée, Revue de Didactologie des languescultures, 116, 477-496.

Marello, C. (1989). Dizionari bilingui. Bologna: Zanichelli.

Mrongovius, C. (1837). Dokładny niemiecko-polski słownik. Dresden: Königsberg in Dresden (edizione consultata).

Nowowiejski, B. (2012). Świat nazw własnych w niemiecko-polskim słowniku Mrongowiusza. Cz. I: Toponimia. Białostockie Archiwum Językowe, 12, 165-182. DOI: 10.15290/baj.2012.12.10. Retrieved from: https://repozytorium.uwb.edu.pl/jspui/handle/11320/1046 (Consultato il 10.04.2019).

Palmarini, L. (2016). Wojciech Meisels: vita e produzione linguistico-letteraria dell'autore del più popolare dizionario bilingue italiano polacco, polacco-italiano. Romanica Cracoviensia, 16, 121-135. DOI: 10.4467/20843917RC.16.011.5932.

Palmarini, L. (2018). La lessicografia bilingue italiano polacca e polacco-italiana, dal 1856 al 1946. Berlin: Peter Lang. DOI:10.3726/b14323.

Rossi, G. (1867). Storia della città di Sanremo. Sanremo: Gandolfo.

Sosnowski, R. (2005). Il dizionario italiano-polacco di Erazm Rykaczewski, alcuni cenni sulla presenza dell'italiano in Polonia nel XIX secolo. Revista de italianística, IX, 173-183.

Tolomeo, C. (1598). Geografia Cioè Descrittione Universale Della Terra. Venezia: Galigani Battista e Giorgio. 


\section{Sitografia}

Jamrozik, E. (2006). Aspetti della lessicografia bilingue. Presentazione del Grande Dizionario italiano-polacco. Retrieved from: http://www.accademiadellacrusca.it/it/scaffali-digitali/ articolo/aspetti-lessicografia-bilingue-presentazione-grande-dizionario-italiano-p (Consultato il 22.04.2019). 